Tiago J. C. Sousa, Vítor Monteiro, José A. Afonso, João L. Afonso

\title{
"Selective Harmonic Measurement and Compensation Using Smart Inverters in a Microgrid with
} Distributed Generation"

IEEE INDIN International Conference on Industrial Informatics, pp.439-444, Porto, Portugal, July 2018.

https://ieeexplore.ieee.org/document/8471952

ISBN: 978-1-5386-4829-2

ISSN: $2378-363 X$

DOI: $10.1109 /$ INDIN.2018.8471952

This material is posted here with permission of the IEEE. Such permission of the IEEE does not in any way imply IEEE endorsement of any of Group of Energy and Power Electronics, University of Minho, products or services. Internal or personal use of this material is permitted. However, permission to reprint/republish this material for advertising or promotional purposes or for creating new collective works for resale or redistribution must be obtained from the IEEE by writing to pubs-permissions@ieee.org. By choosing to view this document, you agree to all provisions of the copyright laws protecting it. 


\title{
Selective Harmonic Measurement and Compensation Using Smart Inverters in a Microgrid with Distributed Generation
}

\author{
Tiago J. C. Sousa ${ }^{1}$, Vítor Monteiro ${ }^{1}$, José A. Afonso ${ }^{2}$, João L. Afonso ${ }^{1}$ \\ ${ }^{1}$ ALGORITMI Research Centre - University of Minho, Guimarães - Portugal \\ ${ }^{2}$ CMEMS-UMinho - University of Minho, Guimarães - Portugal \\ ${ }^{1}$ \{tsousa $\mid$ vmonteiro $\mid$ jla\}@dei.uminho.pt ${ }^{2}$ jose.afonso@dei.uminho.pt
}

\begin{abstract}
This paper presents a frequency domain selective harmonic measurement and compensation using smart inverters (SIs) applied to a microgrid with distributed generation. The compensation current is calculated by a main processing unit (MPU), which controls the compensation action of each distributed generation unit (DGU) connected to the microgrid. A single fast Fourier transform (FFT) is implemented to obtain the frequency spectra of the power grid voltages and the currents consumed by the connected loads. Based on the calculated compensation currents and the availability of the DGUs, the MPU establishes the harmonic orders that each DGU should compensate, aiming to equalize the rms current values among the different DGUs. The proposed approach intends to optimize the DGUs efficiency in the microgrid and, additionally, it allows the connection of the converters to the power grid without the need for a synchronization algorithm. A comprehensive description of the implemented FFT algorithm is performed and, finally, the operation of the proposed scheme is verified by simulation results.
\end{abstract}

Keywords-Microgrid, Distributed Control Systems, Selective Harmonic Compensation, Fast Fourier Transform (FFT).

\section{INTRODUCTION}

Renewable energy sources (RESs) perform a continuously growing role in the present industrialized society, as they contribute to reduce greenhouse gasses emissions and to alleviate the extraction of the pollutant, nonrenewable fossil fuels [1]. Moreover, the integration of RESs in the power grid provides load support during higher demand periods, whereby distributed generation appears as an imminent trend in contrast with the traditional centralized distribution [2][3]. Among the existing types of RESs, wind and solar photovoltaic (PV) represent the main propensity [4][5].

However, RESs are characterized by their intermittence. Reference [6] analyzes the variability and uncertainty of PV energy and management strategies to be considered towards its integration in the power grid. In order to handle the distributed generation intermittence, energy storage systems (ESSs) have a vital role, emerging as a key concern in the future power grids [7]. An economic evaluation of ESSs in a context of RESs is presented in [8], in which the benefits of the former were verified. Besides the problem of intermittence, the interface of RESs with the power grid can origin power quality problems, such as voltage sags and frequency deviations. Being a crucial aspect for the stability of the power grid, power quality must be taken into consideration towards the integration of RESs [9].
As a result of the growing concerns about distributed generation, the paradigms of microgrids and smart grids have emerged and gained attention over the last years [10][11][12], in which power electronics undergoes an essential involvement [13]. Concerning microgrids, these can operate either in islanded mode or in grid-connected mode. Power control strategies for microgrids are investigated in [14] and [15] for islanded operation and in [16] for grid-connected operation. Optimal control strategies from the ESSs point of view can also be considered as presented in [17]. Besides the operation modes, microgrids can be classified accordingly with the type of power, i.e., ac or dc. Dc microgrids are a suitable approach provided that most modern loads rely on dc power, therefore it is possible to eliminate the typical ac-dc conversion and its respective power losses [18]. However, a high-frequency ac approach also offers advantages when compared to traditional line-frequency microgrids [19]. A hybrid microgrid is proposed in [20], addressing the benefits of both ac and dc power.

Power quality issues in microgrids have also been an object of investigation [21]. The compensation of power quality problems is scrutinized in [22], for three-phase four-wire microgrids, and in [23] for high-frequency ac microgrids. Basically, the referred compensators are active power filters of both series and shunt type. Regarding shunt active power filters (SAPFs), the last decades have experienced a considerable research in both hardware topologies and control systems [24]. Several power theories for SAPFs have been proposed and developed, e.g., instantaneous reactive power theory (also called $p-q$ theory) [25], Fryze-Buchholz-Depenbrock power theory [26], current's physical components power theory [27] and synchronous reference frame $(d-q)$ power theory [28]. Among the investigated and proposed theories, time domain power theories are mostly preferred due to the provided fast response and relatively low computational cost. However, the time domain approach is ineffective (and can be even aggravative) towards resonance conditions in the power grid. Harmonic interaction in a context of RESs-based distributed generation is investigated in [29], while [30] and [31] focus on resonance damping in islanded microgrids.

On the other hand, a frequency domain approach allows an individually harmonic measurement, therefore permitting a selective harmonic compensation [32]. Frequency domain power theories apply Fourier analysis, with the fast Fourier transform (FFT) being considered the most efficient class of algorithms to perform such analysis [33]. Nevertheless, FFT calculations require higher computational costs than time 
domain power theories, whereby frequency selective filters can be employed instead [34]. However, this method uses bandpass and bandstop filters that cannot inherently provide satisfactory attenuation without sacrificing the neighbor frequencies, which are narrowly spaced considering power system harmonics.

Besides the compensation domain method, distributed or centralized approaches can be considered. A distributed system of SAPFs was initially proposed in [35], and in [36] is studied the distributed harmonic compensation in a microgrid context. A cooperative control of SAPFs is investigated in [37], in which are presented better results than independent control in terms of harmonic damping. Considering the ongoing necessity of distributed generation and the microgrid paradigm, distributed power quality conditioning is an impending approach for future power grids.

Thereupon, taken into consideration the referred aspects, this paper presents a selective harmonic measurement and compensation using smart inverters (SIs) for a single-phase RESs-based microgrid, where the several connected distributed generation units (DGUs) operate as SAPFs in a distributed manner. A single FFT algorithm is implemented by a main processing unit (MPU) to extract the harmonic content of both power grid voltage and loads current, therefore discarding the use of a synchronization mechanism with the power grid. The MPU evaluates the power availability of each DGU and, as a function of such assessment, defines the harmonic order to be compensated.

The paper is structured as follows: Section II presents the structure of the microgrid from the MPU and DGUs perspective; Section III describes the reference current generation for the SAPF operation, presenting a comprehensive analysis of the implemented FFT algorithm; Section IV depicts the obtained simulation results for the distributed compensation system; lastly, Section $\mathrm{V}$ outlines the main conclusions of the investigation carried out.

\section{MICROGRID STRUCTURE}

This section presents the structure of the DGUs comprising the microgrid, as well as the processing performed by the MPU. A single-line diagram of the microgrid constitution is presented in Fig. 1, where several DGUs, comprised by the respective SI and RES, and different connected loads can be seen. As it can be noticeable, the microgrid is of the grid-connected type.

\section{A. Distributed Generation Units (DGUS)}

Each DGU is comprised by a SI, i.e., a dc-ac power converter connected to the microgrid, and dc-dc and/or ac-dc power converters interfacing the RES with the previously referred converter, depending on the used RES. As it can be seen in Fig. 1, both PV and wind power are considered, whereby the $\mathrm{dc}-\mathrm{dc}$ and/or ac-dc set of power converters operate with the purpose of extracting the maximum power of the RES available at each instant. In the scope of this paper, the power converter topologies are not scrutinized, as well as the operation of the RESs interfacing converters. Therefore, only the operation of the SIs is analyzed from the DGUs point of view. It should be referred that the SIs operate in a current-control scheme, allowing the injection of the extracted energy into the microgrid and, simultaneously, the SAPF operation. Regarding the energy injection, each DGU produces a line-frequency sinusoidal

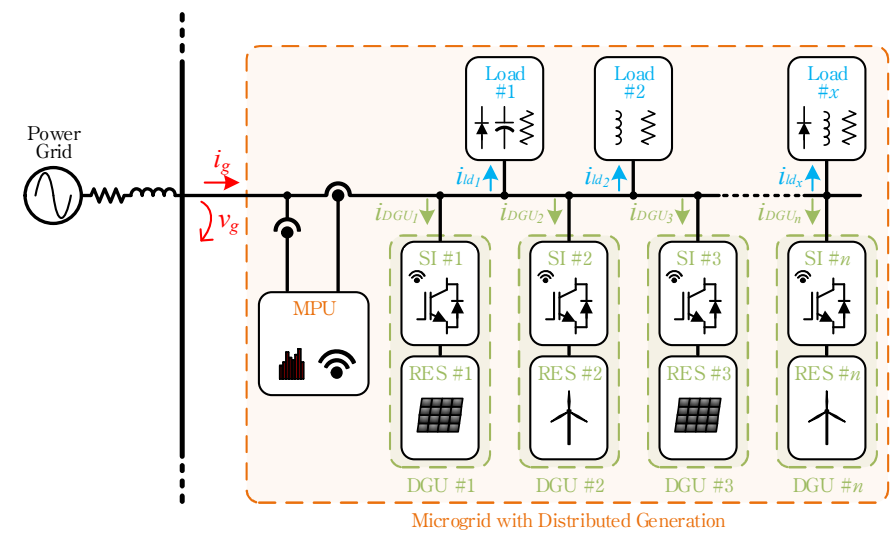

Fig. 1. Structure of the distributed microgrid with $n$ DGUs, the respective RESs and $x$ connected loads.

current with a $180^{\circ}$ phase difference from the grid voltage so that it operates as a power source, aiming for a practically unitary power factor. Considering the SAPF operation, the reference current in the time domain is wirelessly transmitted by the MPU, whereby each DGU must accommodate both current components (fundamental plus harmonics) in its synthesized current so that the two tasks can be properly accomplished.

\section{B. Main Processing Unit (MPU)}

As aforementioned, the MPU calculates the compensation current in the frequency domain. Besides, the MPU monitors the energy injection of each DGU in the microgrid. Since the SAPF operation does not exchange active power with the grid, the harmonic order assignment for each DGU focuses on balancing the rms current along the different DGUs. Afterwards, the MPU communicates the grid voltage and the reference current in the time domain to each DGU, whereby the latter adjusts its total reference current to perform the energy injection into the power grid while compensating the given harmonics.

\section{REFERENCE CURRENT GENERATION}

In this section, the details associated to the calculation of the reference current for the SAPF operation of each DGU are examined. The reference current is attained with a frequency domain method, namely via a complex FFT. Having a high computational burden, the FFT is calculated by the MPU, which commands the operation of the connected DGUs.

\section{A. Real FFT versus Complex FFT}

FFT algorithms are an efficient way of calculating the discrete Fourier transform (DFT). These algorithms can be classified between two types regarding the applied input data: real FFT and complex FFT. The former is appropriate provided that any measured signal in a power system is a real number, whereby this algorithm operates with real numbers, increasing the algorithm computational speed. On the other hand, the complex FFT uses complex numbers as its input data. Although the complex FFT can be used with real numbered inputs, i.e., by setting the input imaginary part to zero, it is less efficient than the real FFT; in fact, the real FFT is an optimized version of the complex FFT for real signals.

However, the complex FFT allows a useful feature towards real signals. Instead of being null, the input imaginary part can be used to place a second real numbered input signal, meaning 


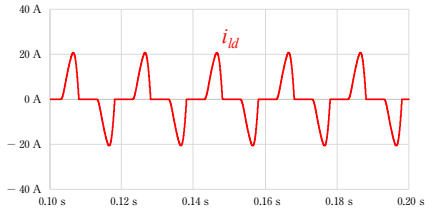

(a)

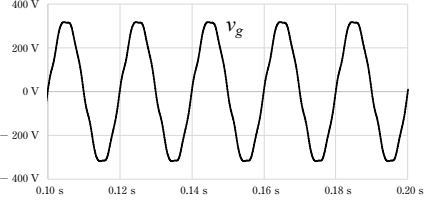

(b)

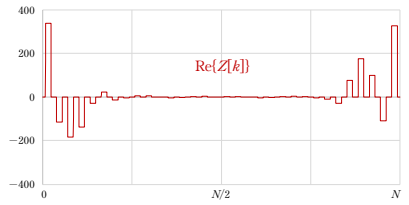

(c)

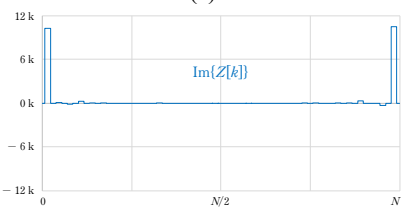

(d)

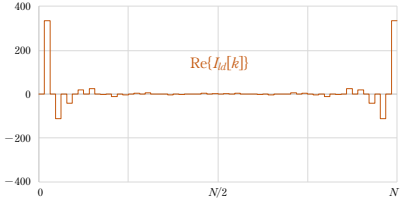

(e)

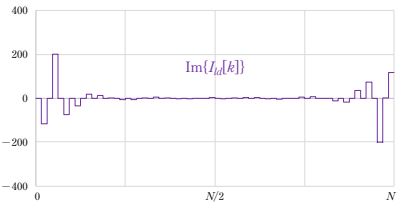

(f)

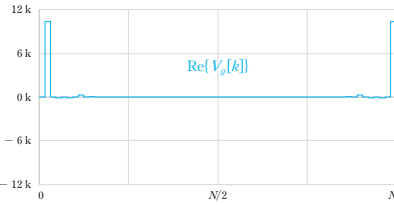

(g)

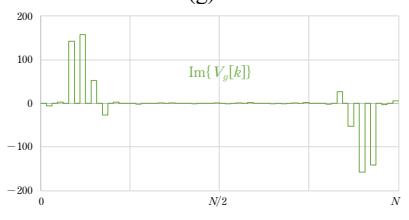

Fig. 2. Dual input complex FFT calculation: (a) Nonlinear load current $\left(i_{l d}\right)$ and (b) power grid voltage $\left(v_{g}\right)$ used as FFT inputs; (c) Real part (Re $\left.\{Z[k]\}\right)$ and (d) Imaginary part $(\operatorname{Im}\{Z[k]\})$ of the FFT of $z[n] ;$; (e) Real part $\left(\operatorname{Re}\left\{I_{l d}[k]\right\}\right)$ and (f) Imaginary part $\left(\operatorname{Im}\left\{I_{l d}[k]\right\}\right)$ of the FFT of $i_{l d}[n] ;(\mathrm{g}) \operatorname{Real}$ part $\left(\operatorname{Re}\left\{V_{g}[k]\right\}\right)$ and

(h) Imaginary part $\left(\operatorname{Im}\left\{V_{g}[k]\right\}\right)$ of the FFT of $v_{g}[n]$

that a single FFT can be applied for two different signals. This is possible due to the existing even and odd symmetries in the frequency spectrum of a signal. Once calculated, the frequency spectrum can be separated in its even and odd parts, allowing the construction of two different spectra that correspond to the individual FFTs of both input signals. This technique allows a processing time reduction of almost a half if more than one signal is intended to be analyzed by an FFT algorithm [38].

\section{B. Complex FFT with Two Input Signals}

As aforementioned, a single complex FFT can be employed to determine the spectra of two real signals. Fig. 2 (a) and Fig. 2 (b) show two distorted power signals, namely a current absorbed by a single-phase diode bridge rectifier with capacitive output filter $\left(i_{l d}\right)$ and the respective supplied voltage $\left(v_{g}\right)$. From both signals, and considering a discrete time implementation, a complex signal $z[n]$ can be assembled. Considering $i_{l d}$ as the real part and $v_{g}$ as the imaginary part, the discrete time domain complex signal $z[n]$ can be attained in accordance with:

$$
z[n]=i_{l d}[n]+j v_{g}[n],
$$

where $j$ represents the complex variable. It should be noted that $z[n]$ is deprived of physical meaning. Afterwards, the time domain signal $z[n]$ can be transposed to the frequency domain by means of the complex FFT, yielding $Z[k]$ :

$$
Z[k]=F F T(z[n])=\operatorname{Re}\{Z[k]\}+j \operatorname{Im}\{Z[k]\},
$$

where $k$ is the discrete frequency index, whose value varies between 0 and $N-1$, with $N$ being the FFT number of points, and $\operatorname{Re}\{Z[k]\}$ and $\operatorname{Im}\{Z[k]\}$ are the real and imaginary parts of the compound $Z[k]$ spectrum, respectively. Both components are shown in Fig. 2 (c) and Fig. 2 (d) and satisfy the following:

$$
\begin{aligned}
& \operatorname{Re}\{Z[k]\}=\operatorname{Re}\left\{I_{l d}[k]\right\}+j \operatorname{Im}\left\{V_{g}[k]\right\} \\
& \operatorname{Im}\{Z[k]\}=\operatorname{Re}\left\{V_{g}[k]\right\}+j \operatorname{Im}\left\{I_{l d}[k]\right\}
\end{aligned}
$$

The objective is to separate each spectrum in order to attain the two spectra $I_{l d}[k]$ and $V_{g}[k]$. As previously referred in this section, the feasibility of a dual input single FFT relies on the symmetry properties of the frequency domain. For a single, purely real input FFT, the real part of the frequency domain presents an even symmetry around the sample $N / 2$, while the imaginary part has odd symmetry. Thus, the following relationships are valid for $k>0$ and $k<N$ :

$$
\begin{aligned}
\operatorname{Re}\{Z[k]\} & =\operatorname{Re}\{Z[N-k]\} \\
\operatorname{Im}\{Z[k]\} & =-\operatorname{Im}\{Z[N-k]\}
\end{aligned}
$$

In a dual input FFT, both types of symmetry are present in the real and imaginary parts. According to (3), $\operatorname{Re}\left\{I_{l d}[k]\right\}$ can be extracted by cancelling $\operatorname{Im}\left\{V_{g}[k]\right\}$, which is possible due to the odd symmetry of the latter. Similarly, $\operatorname{Im}\left\{I_{l d}[k]\right\}$ can be attained from (4) by cancelling $\operatorname{Re}\left\{V_{g}[k]\right\}$ due to its even symmetry. These operations are performed by the following:

$$
\begin{aligned}
\operatorname{Re}\left\{I_{l d}[k]\right\} & =\frac{\operatorname{Re}\{Z[k]\}+\operatorname{Re}\{Z[N-k]\}}{2} \\
\operatorname{Im}\left\{I_{l d}[k]\right\} & =\frac{\operatorname{Im}\{Z[k]\}-\operatorname{Im}\{Z[N-k]\}}{2},
\end{aligned}
$$

whose spectra are presented in Fig. 2 (e) and in Fig. 2 (f), respectively.

For obtaining $V_{g}[k]$, similar operations are used. However, since the time domain signal $v_{g}$ was introduced as an imaginary argument of a complex number, the corresponding spectrum will appear multiplied by $j$, i.e., shifted by $90^{\circ}$. Therefore, instead of cancelling the even component of $\operatorname{Re}\{Z[k]\}$ to obtain $\operatorname{Re}\left\{V_{g}[k]\right\}$, it is necessary to cancel the odd component of $\operatorname{Im}\{Z[k]\}$. Likewise, instead of cancelling the odd component of $\operatorname{Im}\{Z[k]\}$ in order to obtain $\operatorname{Im}\left\{V_{g}[k],\right\}$ it is necessary to cancel the even part of $\operatorname{Re}\{Z[k]\}$ and negate the obtained result. This procedure corresponds to a multiplication by $-j$ (which is a $90^{\circ}$ clockwise rotation) and it is mandatory to be performed in order to obtain the correct phase angles for the frequency components of $v_{g}$. The real and imaginary components of $V_{g}[k]$ are depicted in Fig. 2 (g) and Fig. 2 (h) and are attained according with:

$$
\begin{aligned}
& \operatorname{Re}\left\{V_{g}[k]\right\}=\frac{\operatorname{Im}\{Z[k]\}+\operatorname{Im}\{Z[N-k]\}}{2} \\
& \operatorname{Im}\left\{V_{g}[k]\right\}=\frac{\operatorname{Re}\{Z[N-k]\}-\operatorname{Re}\{Z[k]\}}{2}
\end{aligned}
$$

Once obtained the two separated spectra, it is convenient to transform the rectangular coordinates into polar coordinates, therefore obtaining a value of amplitude and phase for each measured frequency. For a given index $k$ of the frequency spectrum of $i_{l d}$, the corresponding amplitude $\left(A_{I l d}[k]\right)$ and phase $\left(\theta_{\text {Ild }}[k]\right)$ can be attained in conformity with: 


$$
\begin{gathered}
A_{I_{l d}}[k]=\sqrt{\operatorname{Re}\left\{I_{l d}[k]\right\}^{2}+\operatorname{Im}\left\{I_{l d}[k]\right\}^{2}} \\
\theta_{I_{l d}}[k]=\arctan \left(\frac{\operatorname{Im}\left\{I_{l d}[k]\right\}}{\operatorname{Re}\left\{I_{l d}[k]\right\}}\right)
\end{gathered}
$$

The phase angle of the fundamental component of the power grid voltage is the reference for all phase angles for both voltage and current. However, since the algorithm is devoid of synchronization, the calculated phase angle for the power grid voltage fundamental component $\left(\theta_{0}\right)$ is used in the calculations of all remaining angles, making it possible to establish the former as the referential phase. Therefore, for each frequency $k$, the phase angle of its current component $\left(\theta_{I l d}\right)$ will be calculated with respect to $\theta_{0}$.

After obtaining the values of amplitude and phase of each detected frequency for the load current, the control system is capable of generating a reference current for the SAPF operation of the DGUs in a harmonic selective manner. To compensate a given harmonic, the reference current should contain an equal amplitude and a $180^{\circ}$ phase difference to those of the desired harmonic. However, in order to compensate for fundamental reactive power, a different scheme must be applied. Since it is desirable for the power grid to supply only active power to the loads, the DGUs must supply the required reactive power. In the frequency spectrum, this means that the power grid must supply the real part of the fundamental current $\left(\operatorname{Re}\left\{I_{l d}[1]\right\}\right)$, meaning that the compensation current should contain only $\operatorname{Im}\left\{I_{l d}[1]\right\}$, where $k=1$ refers to the fundamental frequency. However, the real and imaginary fundamental current components cannot be used directly because of $\theta_{0}$, since the absolute zero phase coincides with the initial instant of the control algorithm. In this context, the real and imaginary components of the compensation current can be properly attained by means of the following:

$$
\begin{aligned}
& \operatorname{Re}\left\{I_{r e f}[1]\right\}=A_{I_{d d}}[1] \sin \left(\theta_{I_{l d}}[1]\right) \sin \left(\theta_{0}\right) \\
& \operatorname{Im}\left\{I_{r e f}[1]\right\}=A_{I_{l d}}[1] \sin \left(\theta_{I_{d d}}[1]\right) \cos \left(\theta_{0}\right),
\end{aligned}
$$

where $\theta_{\text {Ild }}[1]$ is the phase difference between the fundamental components of $i_{l d}$ and $v_{g}$. Fig. 3 shows a phasor diagram illustrating the referred calculations for the fundamental frequency, where can be seen the load current $\left(\dot{I}_{l d}\right)$, the power grid voltage $\left(\dot{V}_{g}\right)$, the phase angle of the power grid voltage with respect to the FFT algorithm starting $\left(\theta_{0}\right)$, the phase difference between the voltage and current $\left(\theta_{\text {Ild }}\right)$, the reference current for the SAPF operation $\left(I_{r e f}\right)$ and the corresponding real and imaginary components $\left(\operatorname{Re}\left\{I_{r e f}\right\}\right.$ and $\operatorname{Im}\left\{I_{r e f}\right\}$, respectively). It should be noted that this approach dismisses the need of a synchronization algorithm (e.g., phase-locked loop) with the power grid at the expense of an almost negligible extra computation in the FFT algorithm.

\section{Simulation RESUlts}

This section presents the simulation results of the developed compensation scheme for the RESs-based distributed microgrid. The computational simulations were performed with the software PSIM v9.1 from PowerSim.

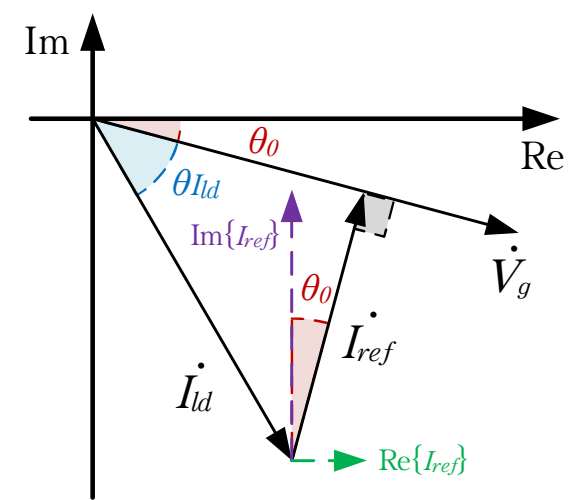

Fig. 3. Phasor diagram representing the calculation of the reference current fundamental component $\left(\dot{I}_{r e f}\right)$ for the SAPF operation.

\section{A. System Parameters}

As referred in Section II, the inner constitution of the DGUs is not discriminated in this paper. Accordingly, the considered power system parameters are the power grid nominal voltage and frequency $(230 \mathrm{~V}, 50 \mathrm{~Hz})$ and the power rating of each DGU, for which a value of $3 \mathrm{~kW}$ (corresponding to a maximum rms current of $13 \mathrm{~A}$ ) was established.

Concerning the FFT algorithm, two main parameters should be evaluated: the sampling frequency $\left(F_{s}\right)$ and the number of points $(N)$. The first parameter defines the maximum measurable frequency, which is $F_{s} / 2$. On the other hand, $N$ is directly related to the number of measurable frequencies, which is $N / 2$. In order to define the spectral resolution of the FFT, both parameters must be selected in consonance. Considering that the microgrid is connected to a power grid with a fundamental frequency of $50 \mathrm{~Hz}$, the desired spectral resolution was chosen to be $50 \mathrm{~Hz}$, which also defines this value as the lowest detectable frequency. Since the spectral resolution is given by $F_{S} / N$ and the optimal values of $N$ in an FFT are powers of two, $F_{s}$ should be selected accordingly. The maximum measurable frequency was chosen to be between $10 \mathrm{kHz}$ and $20 \mathrm{kHz}$, whereby the closest value is attained with $N=512$ and $F_{S}=25.6 \mathrm{kHz}$, which provides a maximum frequency of $12.8 \mathrm{kHz}$ to be measured.

\section{B. Microgrid Operation}

Fig. 4 depicts the operation of four DGUs both before and after the compensation is activated. In the beginning (from $0.02 \mathrm{~s}$ to $0.1 \mathrm{~s}$ ) the compensation scheme is disabled, whereby the DGUs operate with the purpose of injecting energy into the microgrid. As it can be seen in the referred time interval, the

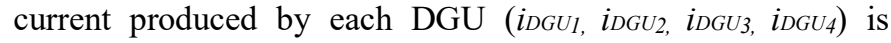
sinusoidal (with a frequency equal to the line-frequency) and in opposite phase with the grid voltage $\left(v_{g}\right)$, showing the contribution of the DGUs to the microgrid. Moreover, the injected currents are insensitive to the distortion of $v_{g}$. It is also noticeable that the DGUs present dissimilar rms current values. On the other hand, the grid current $\left(i_{g}\right)$ is distorted because of the connection of a nonlinear load in the microgrid, which is the load presented in Fig. 2. Despite the distortion, $i_{g}$ contains a large portion of fundamental component, which is a result of the combined energy injection of the DGUs. Due to the same reason, the current $i_{g}$ is in opposite phase with the voltage $v_{g}$. 


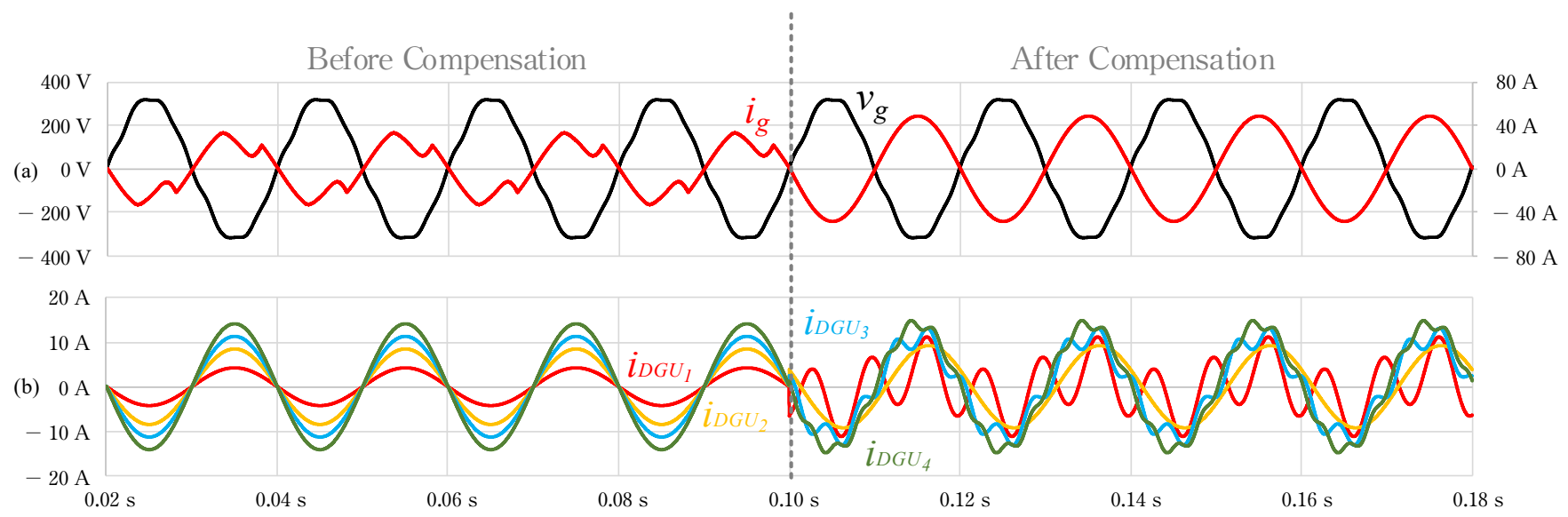

Fig. 4. Simulation results of the proposed scheme with four different DGUs injecting energy into the microgrid before and after performing compensation: (a) Grid voltage $\left(v_{g}\right)$ and current $\left(i_{g}\right)$; (b) Currents produced by DGU $n\left(i_{D G U n}, n=\{1,2,3,4\}\right)$.

At the instant $0.1 \mathrm{~s}$ the compensation is activated, therefore the DGUs start to operate with two purposes: continuing to inject the available energy and compensating the harmonic currents and/or fundamental reactive power required by the connected load. Hence, DGU \#1 compensates the third harmonic $(150 \mathrm{~Hz})$, DGU \#3 compensates the fifth harmonic $(250 \mathrm{~Hz})$ and DGU \#4 compensates the seventh harmonic $(350 \mathrm{~Hz})$. On the other hand, DGU \#2 does not perform harmonic compensation, producing fundamental reactive power instead. In fact, DGU \#2 produces both active and reactive power, as it can be perceived by the phase angle of $i_{D G U_{2}}$, which is neither $180^{\circ}$ nor $-90^{\circ}$. As a result, DGU \#2 provides active power to the microgrid and reactive power for compensating the displacement power factor of the connected load. It can be seen that the coordinate operation of the DGUs after instant $0.1 \mathrm{~s}$ is responsible for the current $i_{g}$ being sinusoidal and in phase opposition with the voltage $v_{g}$.

As aforementioned, the compensation current components are assigned to each DGU as a function of its availability, i.e., in order to distribute the rms current values of each DGU. Table I shows the rms current values of each DGU before and after the compensation starting instant. As it can be noted, the smaller the rms value before the compensation, the larger the increase in the rms value after the compensation. With this strategy, the most supportive DGUs do not succumb to excessive overloads, contributing to the overall efficiency of the microgrid while compensating power quality problems in the current.

TABLE I-RMS CURRENT VALUES OF THE DGUS BEFORE AND AFTER THE COMPENSATION

\begin{tabular}{ccc}
\hline \hline \multirow{2}{*}{ RMS CURRENT } & BEFORE & AFTER \\
& COMPENSATION & COMPENSATION \\
\hline$I_{D G U_{1}}$ & $3.00 \mathrm{~A}$ & $5.90 \mathrm{~A}$ \\
$I_{D G U_{2}}$ & $6.00 \mathrm{~A}$ & $6.53 \mathrm{~A}$ \\
$I_{D G U_{3}}$ & $8.00 \mathrm{~A}$ & $8.22 \mathrm{~A}$ \\
$I_{D G U_{1}}$ & $10.00 \mathrm{~A}$ & $10.04 \mathrm{~A}$ \\
\hline
\end{tabular}

\section{CONCLUSiOnS}

A selective harmonic current compensation scheme using smart inverters (SIs) for a microgrid with distributed generation was presented. The ability to calculate the frequency spectra of two signals in a single fast Fourier transform (FFT) algorithm was demonstrated, discarding the necessity of a synchronization mechanism with the power grid. Besides, the computational cost of calculating a dual input complex FFT is quite similar to that of a single input complex FFT. The FFT was calculated by a main processing unit (MPU), which measures the values of the grid voltages and load currents, as well as the currents injected by the several distributed generation units (DGUs) connected to the microgrid, in order to assess the availability of each one of them. Simulation results were presented to evaluate the selective harmonic compensation for four connected DGUs. It was shown that both harmonic currents and fundamental reactive power can be compensated in a selective manner by means of a single FFT algorithm. The compensation current components were shared among the different DGUs, in order to equalize the rms current of the DGUs as much as possible. With this approach, the resulting power losses within the converters were more evenly distributed along the DGUs, preventing possible malfunctions due to overloads as a consequence of dissimilar current sharing.

\section{ACKNOWLEDGMENT}

This work has been supported by COMPETE: POCI-010145-FEDER-007043 and FCT - Fundação para a Ciência e Tecnologia within the Project Scope: UID/CEC/00319/2013. This work is financed by the ERDF - European Regional Development Fund through the Operational Programme for Competitiveness and Internationalisation - COMPETE 2020 Programme, and by National Funds through the Portuguese funding agency, FCT - Fundação para a Ciência e a Tecnologia, within project SAICTPAC/0004/2015 - POCI - 01-0145FEDER-016434. Mr. Tiago Sousa is supported by the doctoral scholarship SFRH/BD/134353/2017 granted by the Portuguese FCT agency.

\section{REFERENCES}

[1] J. Ansari, A. Gholami, A. Kazemi, and M. Jamei, "Environmental/economic dispatch incorporating renewable energy sources and plug-in vehicles," IET Gener. Transm. Distrib., vol. 8, no. 12, pp. 2183-2198, 2014.

[2] H. Kirkham, D. Nightingale, and T. Koerner, "Energy Management System Design with Dispersed Storage and Generation," IEEE Trans. Power Appar. Syst., vol. PAS-100, no. 7, pp. 3432-3441, Jul. 1981.

[3] M. Olken, "Distributing the load: distributed resources bring generation close to home," IEEE Power Energy Mag., vol. 2, no. 3, pp. 4-6, May 2004. 
[4] M. Milligan, B. Frew, B. Kirby, M. Schuerger, K. Clark, D. Lew, P. Denholm, B. Zavadil, M. O’Malley, and B. Tsuchida, "Alternatives No More: Wind and Solar Power Are Mainstays of a Clean, Reliable, Affordable Grid," IEEE Power Energy Mag., vol. 13, no. 6, pp. 78-87, Nov. 2015.

[5] D. Abbott, "Keeping the energy debate clean: How do we supply the world's energy needs?," Proc. IEEE, vol. 98, no. 1, pp. 42-66, 2010.

[6] A. Mills, M. Ahlstrom, M. Brower, A. Ellis, R. George, T. Hoff, B. Kroposki, C. Lenox, N. Miller, M. Milligan, J. Stein, and Y. Wan, "Dark Shadows," IEEE Power Energy Mag., vol. 9, no. 3, pp. 33-41, 2011.

[7] B. Roberts, "Capturing grid power," IEEE Power Energy Mag., vol. 7 , no. 4, pp. 32-41, Jul. 2009.

[8] N. Li and K. W. Hedman, "Economic Assessment of Energy Storage in Systems with High Levels of Renewable Resources," IEEE Trans. Sustain. Energy, vol. 6, no. 3, pp. 1103-1111, 2015.

[9] X. Liang, "Emerging Power Quality Challenges Due to Integration of Renewable Energy Sources," IEEE Trans. Ind. Appl., vol. 53, no. 2, pp. 855-866, Mar. 2017.

[10] R. H. Lasseter, "MicroGrids," in 2002 IEEE Power Engineering Society Winter Meeting. Conference Proceedings (Cat. No.02CH37309), 2002, vol. 1, pp. 305-308.

[11] C. H. Hauser, D. E. Bakken, and A. Bose, "A failure to communicate: next generation communication requirements, technologies, and architecture for the electric power grid," IEEE Power Energy Mag., vol. 3, no. 2, pp. 47-55, Mar. 2005.

[12] C. W. Gellings, M. Samotyj, and B. Howe, "The future's smart delivery system [electric power supply]," IEEE Power Energy Mag., vol. 2, no. 5, pp. 40-48, Sep. 2004.

[13] B. Kroposki, C. Pink, R. DeBlasio, H. Thomas, M. Simões, and P. K. Sen, "Benefits of Power Electronic Interfaces for Distributed Energy Systems," IEEE Trans. Energy Convers., vol. 25, no. 3, pp. 901-908, Sep. 2010

[14] J de Matos, F e Silva, and L. Ribeiro, "Power Control in AC Isolated Microgrids with Renewable Energy Sources and Energy Storage Systems," IEEE Trans. Ind. Electron., vol. 7, no. 4, pp. 1-1, Jul. 2014.

[15] J. A. P. Lopes, C. L. Moreira, and A. G. Madureira, "Defining Control Strategies for MicroGrids Islanded Operation," IEEE Trans. Power Syst., vol. 21, no. 2, pp. 916-924, May 2006.

[16] Majumder, A. Ghosh, G. Ledwich, and F. Zare, "Power management and power flow control with back-to-back converters in a utility connected microgrid," IEEE Trans. Power Syst., vol. 25, no. 2, pp. 821-834, 2010.

[17] P. Malysz, S. Sirouspour, and A. Emadi, "An optimal energy storage control strategy for grid-connected microgrids," IEEE Trans. Smart Grid, vol. 5, no. 4, pp. 1785-1796, 2014

[18] A. Kwasinski, "Quantitative evaluation of DC microgrids availability: Effects of system architecture and converter topology design choices," IEEE Trans. Power Electron., vol. 26, no. 3, pp. 835-851, 2011.

[19] S. Chakraborty, M. D. Weiss, and M. G. Simoes, "Distributed Intelligent Energy Management System for a Single-Phase High-Frequency AC Microgrid," IEEE Trans. Ind. Electron., vol. 54, no. 1, pp. 97-109, Feb. 2007

[20] Xiong Liu, Peng Wang, and Poh Chiang Loh, "A Hybrid AC/DC Microgrid and Its Coordination Control," IEEE Trans. Smart Grid, vol. 2, no. 2, pp. 278-286, Jun. 2011.

[21] G. Venkataramanan and M. Illindala, "Microgrids and sensitive loads," in 2002 IEEE Power Engineering Society Winter Meeting. Conference Proceedings (Cat. No.02CH37309), 2002, vol. 1, pp. 315-322.
[22] Y. Li, D. M. Vilathgamuwa, and P. C. Loh, "Microgrid Power Quality Enhancement Using a Three-Phase Four-Wire Grid-Interfacing Compensator," IEEE Trans. Ind. Appl., vol. 41, no. 6, pp. 1707-1719, Nov. 2005

[23] S. Chakraborty and M. G. Simoes, "Experimental Evaluation of Active Filtering in a Single-Phase High-Frequency AC Microgrid," IEEE Trans. Energy Convers., vol. 24, no. 3, pp. 673-682, Sep. 2009.

[24] B. Singh, K. Al-Haddad, and A. Chandra, "A review of active filters for power quality improvement," IEEE Trans. Ind. Electron., vol. 46, no. 5 , pp. 960-971, 1999.

[25] H. Akagi, Y. Kanazawa, and A. Nabae, "Generalized Theory of the Instantaneous Reactive Power in Three-Phase Circuits," in International Power Electronics Conference, 1983, pp. 1375-1386.

[26] M. Depenbrock, "The FBD-method, a generally applicable tool for analyzing power relations," IEEE Trans. Power Syst., vol. 8, no. 2, pp. 381-387, May 1993

[27] L. S. Czarnecki, "Orthogonal Decomposition of the Currents in a 3-Phase Nonlinear Asymmetrical Circuit with a Nonsinusoidal Voltage Source,' IEEE Trans. Instrum. Meas., vol. 37, no. 1, pp. 30-34, 1988.

[28] S. Bhattacharya and D. Divan, "Synchronous frame based controller implementation for a hybrid series active filter system," in IAS '95. Conference Record of the 1995 IEEE Industry Applications Conference Thirtieth IAS Annual Meeting, 1995, vol. 3, pp. 2531-2540.

[29] J. H. R. Enslin and P. J. M. Heskes, "Harmonic Interaction Between a Large Number of Distributed Power Inverters and the Distribution Network," IEEE Trans. Power Electron., vol. 19, no. 6, pp. 1586-1593, Nov. 2004.

[30] J. He, Y. W. Li, D. Bosnjak, and B. Harris, "Investigation and Active Damping of Multiple Resonances in a Parallel-Inverter-Based Microgrid," IEEE Trans. Power Electron., vol. 28, no. 1, pp. 234-246, Jan. 2013.

[31] Xiongfei Wang, F. Blaabjerg, and Zhe Chen, "Autonomous Control of Inverter-Interfaced Distributed Generation Units for Harmonic Current Filtering and Resonance Damping in an Islanded Microgrid," IEEE Trans. Ind. Appl., vol. 50, no. 1, pp. 452-461, Jan. 2014.

[32] W. M. Grady, M. J. Samotyj, and A. H. Noyola, "Survey of active power line conditioning methodologies," IEEE Trans. Power Deliv., vol. 5, no. 3, pp. 1536-1542, Jul. 1990.

[33] J. W. Cooley and J. W. Tukey, “An algorithm for the machine calculation of complex Fourier series," Math. Comput., vol. 19, no. 90, pp. 297-297, May 1965.

[34] M. Illindala and G. Venkataramanan, "Frequency/Sequence Selective Filters for Power Quality Improvement in a Microgrid," IEEE Trans. Smart Grid, vol. 3, no. 4, pp. 2039-2047, Dec. 2012.

[35] P.-T. Cheng and T.-L. Lee, "Distributed Active Filter Systems (DAFSs) A New Approach to Power System Harmonics," IEEE Trans. Ind. Appl., vol. 42, no. 5, pp. 1301-1309, Sep. 2006.

[36] Y. W. Li and J. He, "Distribution System Harmonic Compensation Methods: An Overview of DG-Interfacing Inverters," IEEE Ind. Electron. Mag., vol. 8, no. 4, pp. 18-31, Dec. 2014.

[37] P. Jintakosonwit, H. Fujita, H. Akagi, and S. Ogasawara, "Implementation and performance of cooperative control of shunt active filters for harmonic damping throughout a power distribution system," IEEE Trans. Ind. Appl., vol. 39, no. 2, pp. 556-564, Mar. 2003.

[38] S. W. Smith, The Scientist and Engineer's Guide to Digital Signal Processing, 2nd ed. San Diego, California: California Technical Publishing, 1999. 